\title{
Die Verausländerung
}

\author{
Erhard Taverna
}

«Dieses Gefühl der geistigen und kulturellen Schicksalsverbundenheit gilt es zu wecken und zu fördern. Eine Voraussetzung dafür ist, dass wir uns innerlich näher treten, dass wir uns im besonderen mehr als bisher Rechenschaft ablegen über den Aufbau und die Lebensäusserungen der Volksgemeinschaft, der wir angehören. Wer kann uns darüber besser Auskunft geben, als eben diese Volksgemeinschaft selbst? Hören wir, was sie, die Gesamtheit von vier Millionen, uns zu sagen hat.» Was das Volk zu sagen hat, fasst der damalige Direktor des Eidgenössischen Statistischen Amtes auf dreissig folgenden Seiten zusammen.

\section{9}

Als demographische Probleme nennt er vor allem die Landflucht und Verstädterung, die «Verausländerung», die sprachliche und konfessionelle Mischung, den Geburtenrückgang und die Überalterung. Es sind die gleichen Themen, die das gleiche Amt, rund 60 Jahre später, wieder auflisten wird. Angesichts der totalitären Bedrohung, kurz vor einem neuen Krieg, interpretiert er seine Zahlen im Sinne der zeitgemässen «geistigen Landesverteidigung». Zwar begrüsst er den Ausländerrückgang um die Hälfte (8,7\% 1930 im Vergleich zu 14,7\% 1910), misstraut aber dem «echt schweizerischen Gesinnungswandel» der allzu viel Eingebürgerten (128 000). Ihn bedrückt die bange Frage, ob die 78000 Schweizer, die seit 1910 eine Ausländerin geheiratet haben, ihre Kinder «im guten Schweizersinn» erziehen, oder sich gar landesfremde Anschauungen zu eigen machen. Da ist es eine Beruhigung zu wissen, dass die Hälfte dieser Frauen im Lande geboren wurde. Da er den Müttern mehr erzieherischen Einfluss zugesteht als den Vätern, fallen die staatspolitischen Bedenken gegenüber den 32000 Schweizertöchtern, die 1910-1937 einen Ausländer ehelichten, weniger ins Gewicht. Zudem weiss er, dass «sie durch Elternhaus, Freunde und Verwandte im angestammten Volk verankert bleiben und ihr Erbgut auch im neuen Milieu treu verwalten». Das ungleiche Zahlenverhältnis wird durch das inländische Überangebot an ausländischen Heiratskandidatinnen begründet, die zudem den Schweizer Mann durch ihren hauswirtschaftlichen Beruf verlocken würden.
Ähnlich bedenklich ist der Geburtenrückgang auf 125 Kinder pro 1000 verheiratete Frauen. Die Bevölkerung wird bereits in der dritten Generation auf die Hälfte absinken und die gebärfreudigeren Katholiken werden den protestantischen Nachwuchs überholen. Die Grafik der Alterspyramide hat sich seit 1900 drastisch geändert. Der gesunde Altersaufbau unterliegt einem Zerstörungsprozess. Immer mehr Greise stauen sich in den oberen Altersklassen, was unausweichlich zu einem «Dammbruch» führt. «Schwer lastet auf unserem Geschlecht diese Hypothek des Todes.» 1960 wird die Schweiz 100000 Einwohner weniger zählen als heute, 1970 könnten es gar mehrere 100000 sein. Die Armee verliert Soldaten, die Alterasyle füllen sich, die Schulstuben verwandeln sich in Pfrundstuben, die Bauern werden ihre Milch nicht mehr los, die konservative Weltanschauung gewinnt wieder breiten Raum.

\section{3}

«Informationen aus der Demografie», nennt dasselbe Amt seinen Bericht 64 Jahre später. Was 1939 als «Gedränge und Verstädterung» beschrieben wurde, hat sich inzwischen in flächendeckenden Agglomerationen fast verdoppelt. Geändert hat sich die Terminologie, die Schwerpunktthemen sind fast identisch: die abnehmende Geburtenhäufigkeit (1,22 Kinder je Schweizerin, 1,88 Kinder je Ausländerin), die «Gesellschaft des langen Lebens» und die Ausländer, die von 1946 bis 1995 mindestens 35\% zum Bevölkerungswachstum des Landes beigetragen haben. Bei den 1960 Geborenen beträgt dieser Zuwachs sogar 60\%. Chancen und Risiken werden, wen wundert's, anders beurteilt. Die Ökologie macht Sorgen, die Finanzierung der Alterspflege und damit im Zusammenhang der Generationenkonflikt aufgrund der zunehmend auseinanderdriftenden Steuerbilanz. Weiter sind es die Erwerbsquote, die fehlenden Angebote zugunsten der benötigten Erwerbstätigkeit der Frauen und das Defizit an jungen Arbeitskräften. Es sind die Zunahme des Verkehrs, die Abnahme der Landwirtschaftszonen bei steigendem Druck auf die verfügbare Wohnfläche. Lebenslanges Lernen ist angesagt, die Grundausbildung wird in Frage gestellt, eine gezielte Migrationspolitik 
soll entlasten, kohärente, koordinierte und nachhaltige Strategien ein Gleichgewicht herstellen. Das Ganze sollte rechtzeitig umgesetzt und der Bevölkerung frühzeitig mitgeteilt werden, «damit diese ausreichend darauf vorbereitet ist». Der Bericht spricht von einem «window of opportunity» bis 2010 und fasst die Handlungsbereiche und Akteure tabellarisch zusammen: die öffentliche Hand ist gefordert, die Unternehmen, die Haushalte und Individuen. Man darf gespannt sein auf den nächsten Bericht. In 60 Jahren.

- Eidgenössisches Statistisches Amt. Wir als Viermillionenvolk. Zur Schweizerischen Landesausstellung 1939. Bern; 1939.

- Bundesamt für Statistik. Informationen aus der Demografie. Chancen, Risiken und Herausforderungen der demografischen Entwicklung. Neuenburg 2003;1.

\section{Angst und Faszination in der Balintarbeit}

C. Föhn

Mit diesem Referat wurden die etwa 50 Teilnehmenden der 41. Silser BalintStudienwoche (15.-21. September 2002 in Sils im Engadin) begrüsst und in eine intensive Arbeitswoche eingeführt. Die Teilnehmerinnen und Teilnehmer sind etwa zu einem Drittel Ärzte mit unterschiedlicher Spezialisierung (Internisten, Allgemeinmediziner, Pädiater, Zahnärztinnen u.a.), zu einem Drittel Psychiater und zu einem Drittel Ärzte, die neben ihrer somatischen Praxistätigkeit psychotherapeutisch arbeiten (darunter vor allem Kollegen aus Deutschland). Dieses Jahr nahmen kaum psychologische Psychotherapeuten teil und leider keine Teilnehmer aus Pflege, medizinisch-therapeutischen Berufen und Seelsorge. Viele der Psychiaterkolleginnen und -kollegen kamen zur Aus- und Weiterbildung in Balintgruppenleitung nach Sils. Die Fallbesprechungen fanden morgens in der Grossgruppe und nachmittags in der Kleingruppe statt (Grossgruppe: eine Innengruppe von etwa acht Teilnehmern diskutieren einen Fall in Anwesenheit der Aussengruppe [alle Teilnehmer], die vom Leiter gelegentlich ins Geschehen einbezogen wird). Abends wurden in einer offenen Diskussionsrunde die aufgelaufenen Themen zur Sprache gebracht.

Die Referentin selber gehört dem Leiterteam der Silser Woche an, ist Fachärztin für Psychiatrie und Psychotherapie, arbeitet in eigener Praxis und leitet eine interdisziplinäre Balintgruppe.

Korrespondenz:

Dr. med. Clara Föhn

Walserstrasse 10

CH-9014 St.Gallen

Tel. 0712780350

Fax 0712780302

E-Mail: clara.foehn@hin.ch
Beim Überlegen, womit ich Sie zu unserem Seminar begrüssen könnte, habe ich kurzerhand beschlossen, eine Teilnehmerin meiner Balintgruppe zu fragen, was sie denn hören möchte als Einführung zu einem solchen Seminar. Sie hat geantwortet, sie möchte die Versicherung haben, dass nichts Schlimmes passiert, wenn man sich hier in die Innengruppe setzt und einen Fall vorstellt. Und - sie möchte eigentlich wissen, was mich nach so langer Zeit immer noch interessiere an dieser Form der Arbeit.
Sehr zufrieden damit habe ich über die Fragen nachzudenken begonnen:

1. Was geschieht hier eigentlich und warum macht das Angst? und

2. Was fasziniert mich an dieser Arbeit immer noch?

Je mehr ich darüber nachdenke, desto deutlicher wird mir, dass das Angstmachende und das Faszinierende etwas miteinander zu tun haben müssen:

Zum Angstmachenden: Ich kann mich gut erinnern, dass es Angst macht: Als ich vor über 20 Jahren die ersten Male - als Psychiatrieassistentin - hierhergekommen bin, habe ich mich nie in die Innengruppe zu setzen gewagt und in der Aussengruppe nie das Maul aufgemacht. Ich bin zwar manchmal fast geplatzt, weil in meinen Augen die Gruppe das Wichtigste einfach nicht sehen wollte. Ich konnte aber meinen Teil nicht einbringen, ihn somit kaum in Beziehung setzen mit dem ganzen Geschehen, und blieb in gewissem Sinn draussen. Trotzdem blieb ich dabei, war trotzdem fasziniert, konnte immer mehr Zusammenhänge erfassen und wurde - nach vielen Jahren immer aktiverer Teilnahme an Balintgruppen - paradoxerweise sogar Balintgruppenleiterin.

Was macht denn Angst? Ich setze mich in den Innenkreis, erzähle von einer Begegnung mit einem Patienten, bei dem mir auf irgendeine Weise nicht wohl gewesen ist. Der Patient, von dem ich erzähle, beschäftigt mich, sonst käme er 
mir nicht in den Sinn, er hat ungute oder lästige Gefühle geweckt. Ich weiss, dass es auch etwas mit mir zu tun hat, ich bin irgendwie getroffen, komme nicht los davon. Wenn ich da referiere, stehe ich nicht über der Sache, verstehe es selber noch nicht und weiss auch nicht, was ich bei der Gruppe, beim Leiter, bei der Aussengruppe auslöse. Vielleicht ähnliche Gefühle wie der Patient bei mir? Wie unangenehm! Werde ich lächerlich gemacht? Entlarve ich mich als inkompetent? Wird man mich nachher noch ernst nehmen? Schliesslich habe ich einen guten Ruf zu verlieren!

Ich erzähle trotzdem, was mir von der Begegnung in Erinnerung ist. Das Erzählte wird aufgenommen von der Gruppe, der Faden wird weitergesponnen, Phantasien tauchen auf, werden in Frage gestellt, ausgemalt, überraschende Aspekte dazugestellt. Plötzlich merkt man als Referent, dass man - man versteht es selber nicht zuvor wichtige, augenfällige Seiten des Patienten oder der Beziehung nicht gesehen hat. Die unangenehmen Gefühle erhalten einen Sinn: sie sind Teil eines Beziehungsmusters, das in der Gruppe plötzlich sichtbar wird. Ein Beziehungsmuster wird sichtbar, das unsere Erwartung, mit dem Patienten gut zusammenarbeiten zu können, gestört hat. Etwas hatte zuvor fast gewaltsam von einem Besitz ergriffen, so dass man nicht mehr frei war zu sehen, zu denken und seine Arbeit zu tun. Mit Hilfe der Gruppe gelingt es einem, etwas Distanz zu finden, etwas wahrzunehmen, sich zu befreien aus der Verstrickung und - im Idealfall - vielleicht zu realisieren, dass einem der Patient auf diese Weise etwas spüren lässt, was er sonst nicht ausdrücken kann, was er selber nicht erkennt. Vielleicht lässt er einen so etwas spüren von dem, was sein Leben wirklich schwierig macht, etwas von dem, was er vermutlich auch sonst mit Menschen macht, mit Menschen, von denen er etwas möchte, die ihm nahestehen, oder von denen er sich abhängig fühlt. Dann wird klar, dass die unangenehmen Gefühle, die einen dazu gebracht haben, sie in der Balintgruppe vorzustellen, nicht nur eine Störung darstellten, die es zu beseitigen gilt, sondern auch eine wichtige Wahrnehmung möglich machen, die helfen kann, unbewusste, averbal geäusserte Schwierigkeiten von Patienten zu verstehen; Schwierigkeiten, die vermutlich auch deren Alltagsbeziehungen vergiften. Die Störung, das oft so enorm Unangenehme, kann also gerade ein tieferes Verständnis ermöglichen.

Ausgehend von der Angst sind wir beim Faszinierenden gelandet: Unangenehme, zuweilen qualvolle Empfindungen können Schlüssel zum besseren Verständnis werden.
Ein Beispiel aus meiner Praxis, das diese Verwandlung konkreter machen soll:

Es ist die allererste Patientin in meiner Praxis, nachdem ich zuvor viele Jahre als Konsiliarpsychiaterin am Spital gearbeitet habe: Sie erreicht, dass ich nach der Konsultation am liebsten meine Praxis wieder schliessen und den Beruf an den Nagel hängen möchte. Ich verstehe, was passiert ist, erst nach einer Besprechung in meiner Intervisionsgruppe, die «balintoid» arbeitet. Hier kann ich mir vergegenwärtigen, was zwischen mir und der Patientin geschehen ist. Ich schaffe das erst in der Gruppe, weil das Nachdenken über diese Stunde dermassen unangenehm war, dass ich es immer wieder gelassen habe. Ich kenne die über 60jährige Patientin von meiner Arbeit am Spital. Ich habe sie dort zweimal gesehen. Ich habe die Diagnose einer Depression mit Panikstörung gestellt. Ich weiss von ihr, dass sie als Kind über lange Zeit misshandelt und missbraucht worden ist - ihre eigene Mutter spielte dabei eine zentrale Rolle. Sie hat mir diese Geschichte in der ersten Stunde anvertraut und verlangt, dass ich das nicht in ihre KG schreibe, weil das ausser ihrem früheren Psychiater und mir niemand wissen dürfe. Sie wollte unbedingt weiter in meine Behandlung kommen. Die Patientin, Mutter von inzwischen mehreren erwachsenen Kindern und Grossmutter vieler Enkel, tritt normalerweise als eine mädchenhafte, strahlend lächelnde Person auf, die sich gleichzeitig unterwürfig und devot benimmt; sie bietet allen ihre Hilfe an, klappt aber von Zeit zu Zeit einfach zusammen und braucht dann notfallmässig selber Hilfe (sie hat akute Hyperventilationsanfälle). Nur in der allerersten Stunde hat sich die Patientin anders verhalten, echt, so dass ich mich gut in sie einfühlen konnte. In der zweiten Stunde im Spital und später in der ersten Stunde in der Praxis war sie unnatürlich liebenswürdig, meine Praxis in den höchsten Tönen rühmend und mir dankend, dass sie kommen dürfe, usw. Sie bat mich dringend um Hilfe, und zwar in Form von einem «Pilleli», damit sie wieder arbeiten könne. Das letzte Pilleli, das sie im Spital auf meine Veranlassung erhalten habe (ein Antidepressivum), habe sofort Wunder gewirkt, es sei ihr danach wunderbar gegangen. Aber später (nach ein paar Wochen) habe das Pilleli dann allerdings geschadet, sie habe es absetzen müssen. Sie müsse jetzt ein anderes haben. Alle meine Versuche, mit der Frau auf einer anderen Ebene ins Gespräch zu kommen, scheitern. Sie will nur so ein «Scheisspilleli», von dem ich überzeugt bin, dass es ihr nichts bringt. Aber ich gebe ihr eines. Am Ende der Stunde stellt sie etwas überlegen 
lächelnd fest, sie hoffe, sie habe mich nicht zu sehr belastet, so dass ich meinen schweren Beruf weiter ausüben könne. Ich bin hilflos wütend und fühle mich missbraucht, schäme mich, dass ich mich habe hinreissen lassen, ihr in der hilflosen Wut tatsächlich eine Pille zu verschreiben, die dann auch wirklich nichts genützt hat. Zudem bin ich konsterniert, dass sie meinen Gefühlszustand so genau durchschaut hat. Ich hätte am liebsten den Laden wieder geschlossen. In der Diskussion mit den Kollegen hatte ich mein Aha-Erlebnis: Zwischen uns ging es um Macht und Machtmissbrauch. Sie vermittelte mir die Gefühle, die sie mit sich seit Jahrzehnten herumtragen muss und die sie auch nicht hochkommen lassen will, weil sie unerträglich sind: Die Gefühle eines hilflosen, missbrauchten Kindes, dass das machen musste, was man von ihm verlangte. Mit dieser Erkenntnis ging ich einigermassen neugierig in die nächste Sitzung. Ich entschloss mich, die Patientin in allen ihren Entscheiden zu unterstützen, und bestätigte, dass sie in ihrem Leben gelernt habe, sich selber zu helfen, und dass ich sie dabei allenfalls unterstützen könne; ich versuchte, ihre Autonomie und Kompetenz absolut zu respektieren; damit erreichte ich zumindest, dass ich Distanz bekam und im Täter-Opfer-Muster nicht weiter mitspielte. Symptomatisch ging die Krise vorbei wie schon oft - und wahrscheinlich nicht durch meine Hilfe. Ich bot der Patientin an, quasi prophylaktisch weiter im Gespräch zu bleiben, was sie nach einiger Überlegung annahm. Bereits zwei Stunden später berichtete sie vom Auftauchen einer unbändigen Wut über ihre vor vier Jahren verstorbene Mutter und darüber, dass sie immer noch Mutters fürchterlich nach Kampfer stinkende Truhe neben ihrem Bett stehen habe. Sie warf die Truhe raus und fühlte sich bärenstark dabei. Damit ist sie natürlich noch nicht geheilt. Sie musste diese Gefühle auch vorübergehend büssen (mit Pseudohalluzinationen, in welchen sie ihre wütende Mutter hinter sich sah); es ist aber etwas Stimmiges passiert, dem ich nur Platz geben konnte dank dem Wahrnehmen meiner eigenen gefühlsmässigen Reaktion in der Balintgruppe. Wenn ich nicht darüber hätte nachdenken können, hätte ich die Frau mit unterdrückter Wut und Ablehnung freundlich herablassend behandelt, und damit hätte ich mich - so befürchte ich - mit der Patientin im alten Muster im Kreis gedreht, und alles wäre gleich geblieben.

Ich habe hier ein Beispiel gebracht als Illustration, wie die Störung der Beziehung sich in den Schlüssel zum Verständnis verwandeln kann - im Moment, in welchem ich die Angst und die Abscheu, hinzuschauen, überwinde und mich damit einem Prozess überlasse, von dem ich nicht zum voraus weiss, wohin er führen wird.

Ich habe ein psychiatrisches Beispiel gebracht. Wir werden wahrscheinlich hier einige Fälle aus der psychiatrischen und aus der psychotherapeutischen Behandlung hören. Dadurch kann eine weitere Form von Angst oder vielleicht auch Befürchtung auftauchen, nämlich als Hausarzt, als Spezialist für Organkrankheiten nun auch noch Psychotherapie machen zu müssen - und damit eher noch mehr belastet als entlastet zu werden. Nun ist es ja Tatsache, dass solche Verwicklungen, solche Beziehungsstörungen überall entstehen können, vor allem aber in Beziehungen, in denen Hilfe, Abhängigkeit, Nähe (Nähe in Phantasie und Erwartung oder in Realität) eine Rolle spielen. Also in jedem Spital, in jeder Hausarztpraxis, in jedem Helferoder Abhängigkeitssystem. Diese Beziehungsstörungen belasten einen sehr. Sie verhindern nämlich, dass man seine Arbeit zur eigenen Zufriedenheit machen kann. Wenn es gelingt, die Störung zu erkennen und einzuordnen, verschafft dies Luft und Spielraum. Dies ist das Ziel der Balintgruppenarbeit. Was wir dann weiter damit machen, ist berufsspezifisch verschieden. Psychotherapeuten/Psychoanalytiker werden den Anspruch haben, sich direkt mit der Beziehungsstörung $\mathrm{zu}$ befassen und damit $\mathrm{zu}$ arbeiten - eben psychotherapeutisch tätig zu werden. Der Hausarzt (und im obigen Fall auch ich als Psychiaterin) wird versuchen, aus der Falle auszusteigen (im Beispiel aus der OpferTäter-Falle, wo hilflose Opfer zu Tätern und Täter zu wütenden Opfern werden), aussteigen, um zumindest nicht zu schaden und die Störung nicht noch weiter zu verstärken. Der Arzt wird dann auch klären wollen, was eigentlich der Auftrag des Patienten ist (ist es die Abklärung der Atemnot und Information darüber, ist es auch eine allfällige organbezogene Behandlung, oder möchte er mit ihm zusammen die Hintergründe und Risikofaktoren solcher Beschwerden ausleuchten, oder sucht er einen Verbündeten gegen die Welt oder Bestätigung, dass er hilfloses Opfer ist, das von der Täterumgebung krank gemacht worden ist?). Der Hausarzt (stellvertretend für alle Helfer gemeint) wird sich erlauben, sich zu überlegen, ob er den Auftrag übernehmen kann oder nicht oder wie weit - und wird sich nicht in blindem Eifer an die Erfüllung vermeintlicher oder unerfüllbarer oder sogar schädlicher Aufträge machen. Denn das wäre der direkte Weg zurück in die Falle.

Ich bin mir inzwischen sicher, dass wir alle, Psychotherapeuten und Hausärzte, so erfahren wir auch immer sein mögen, uns immer wieder 
in solchen Beziehungsfallen wiederfinden, ohne es rechtzeitig zu merken, und froh sind um die Hilfe, dies zu erkennen. Wir werden nie gefeit sein davor. Wir werden Erfahrung sammeln können, solche Muster rascher zu erkennen und damit auch umzugehen. Die Gewalt aber, mit der man zuweilen in solche Muster hineingezogen wird, ist enorm - das erschüttert mich immer wieder -, am meisten, wenn's mir selber passiert.

Wir sind bei einer weiteren Befürchtung angelangt, die angesichts der Balintarbeit auftaucht, nämlich, ob dies nicht Zeitverschwendung ist und ob das überhaupt etwas nützt. Da besuchen Fachleute jahrelang Balintgruppen und gehen immer und immer wieder nach Sils. Können sie es denn immer noch nicht? Das ist doch nicht effizient! Und dann sagt sogar noch eine Leiterin, dass sie selber immer noch dieselben Fehler macht! Auf dieser ökonomischen Ebene zu argumentieren, ist mir zwar nicht so vertraut, aber ich habe eine stolze Antwort darauf. Ich wage nämlich zu behaupten, dass die Balintgruppenmethode eine gute Möglichkeit ist, ineffiziente Behandlungen effizienter zu machen. Ineffizient ist eine Behandlung z. B. dann, sagen viele Artikel in der Fachpresse und in Tageszeitungen, wenn der Patient keine «Compliance» zeigt, wie man so schön sagt, d. h., wenn er die teuren Tabletten wegschmeisst oder anders einnimmt als besprochen, die guten Ratschläge zur Lebensführung nicht befolgt usw. - und dies alles dem Arzt nicht mitteilt, so dass dieser mit seiner Behandlung ins Leere läuft. Die Zeit vergeht, die Kosten steigen, das Problem ist nicht gelöst, der Arzt wird gewechselt, die gutgemeinte Psychotherapie läuft sich langsam tot. Wahrscheinlich tun wir dann als Helfer eines nicht mitarbeitenden Patienten etwas, das dieser nicht will, wodurch dieser sich nicht verstanden fühlt. Jedenfalls ist die Effizienz bestimmt in Frage gestellt, wenn wir den wirklichen Auftrag des Patienten nicht erkennen. Die Compliance dürfte wesentlich besser sein, wenn es gelänge, die Störungen zu erkennen und für ein besseres Verständnis zu nutzen, oder wenn es doch zumindest gelänge, im zerstörerischen Muster nicht mitzuspielen. Und genau hier bietet sich die Balintgruppenarbeit als effizientes Werkzeug an.

In Anlehnung an ein hübschen Büchlein («The Doctor's Communication Handbook» von Peter Tate) ist man als Arzt dann effektiv, wenn man rasch den Auftrag des Patienten erkennt, wenn möglich den wirklichen - nicht nur den vermeintlichen; wenn man klärt, ob man den Auftrag annehmen kann und will oder allenfalls nur Teile davon, und den Weg bespricht, wie man den Auftrag abwickeln kann. Dabei ist es für die Verständigung wesentlich, dass man dieselbe Sprache spricht - klärt, ob man sich gegenseitig wirklich verstanden hat, und ob man gemeinsam ein Arbeitsbündnis eingehen will. Das sind Regeln der Kommunikation - nicht Psychoanalyse. Diese Anforderungen (Auftrag klären usw.) sind in einer ungestörten Beziehung selbstverständlich und relativ einfach zu erfüllen. Wenn dies klappt, hat man als Helfer vermutlich Spass an seiner Arbeit, zumindest erscheint sie einem sinnvoll. Das Arbeitsbündnis ist ungestört und fruchtbar, auch wenn die Arbeit schwer und belastend sein kann; auch wenn es um bedrückende Fakten geht, trägt man es doch gemeinsam, man tut die Arbeit gemeinsam, jeder seinen Teil. Der Patient seinerseits glaubt mit Sicherheit, er habe einen guten Doktor, sogar dann, wenn er an seiner Krankheit sterben sollte, und wahrscheinlich sogar dann, wenn dem Arzt Fehler unterlaufen sollten. Wenn die Beziehung hingegen gestört ist, kann es schwierig sein, den wirklichen Auftrag des Patienten zu erkennen, man fühlt sich sofort unter Druck, in bestimmter Weise zu handeln und sich zu verhalten. Man verliert die Freiheit zu klären, was Auftrag, was eigene Möglichkeit ist, sogar ob man sich verstanden hat und ob man überhaupt zusammenarbeiten will und auf welchem Weg.

Natürlich kann es zu Störungen kommen, nicht nur durch den Patienten, sondern auch durch den Helfer, z.B., wenn dieser übermüdet ist, sich als Opfer seiner Arbeit fühlt. Dann sehen alle Patienten gleich aus; er sieht überall dasselbe; er mag nicht mehr wirklich hinschauen und hinhören. Er verliert seine Neugierde und Offenheit.

Ich möchte zur Illustration zwei banale Beispiele erzählen: Eine Frau um die 50 kommt wegen Kreuzschmerzen zum Hausarzt. Dieser hört das Wort Kreuzschmerz, denkt: Aha, sie will ihren Kreuzschmerz behandelt haben; er beginnt gleich auszufragen, wann-wie-wo-wohin und veranlasst vielleicht sogar ein bisschen «contre cœur» ein Röntgenbild (weil er denkt, sonst fühle sich die Frau nicht ernstgenommen) und verschreibt ein Antirheumatikum. Er verabschiedet die Frau und freut sich über den speditiven Ablauf der Sprechstunde. Die Frau ihrerseits kommt in ihrer Erzählung nicht weiter als bis zu «Kreuzschmerz» - denkt dann: «Ja, wenn der Arzt das so wichtig nimmt, wird es vielleicht schon was Ernstes sein». Die Schmerzmittel passen ihr nicht ganz, aber es geht ja alles so schnell und unversehens ist sie wieder draussen. Eigentlich - kommt ihr viel später wieder in den Sinn - hätte sie darüber sprechen wollen, dass ihr die 
Pflege der Schwiegermutter im selben Haus langsam zu viel werde und wollte mit dem Arzt besprechen, was er dazu meine, was man tun könnte etc. Sie hat es während der Konsultation völlig vergessen. Sie denkt später, der Arzt wollte ihr vielleicht indirekt sagen, sie solle sich nicht so anstellen, sie könne ja ein Schmerzmittel nehmen, dann halte sie das mit der Schwiegermutter schon noch aus. Eskalation der Missverständnisse. Diese Frau war auch meine Patientin - ihr Anteil an den Missverständnissen lag sicherlich auch darin, dass sie wegen Schuldgefühlen nicht zu sagen wagte, die Schwiegermutter sei ein Problem, sondern vor sich und dem Hausarzt die Kreuzschmerzen als Rechtfertigung brauchte.

Ein anderes Beispiel: Eine Frau um die 50 kommt zum Gynäkologen wegen Menorrhagien. Er stellt Myome fest und empfiehlt die Hysterektomie. Die Patientin ist medizinisch interessiert, hat schon viel über die Folgen von Hysterektomien gelesen und möchte wissen, wie das mit der sexuellen Empfindungsfähigkeit sei, wenn die ganze Gebärmutter mit Muttermund entfernt werde. Sie fragt nun, ob man nicht den Muttermund belassen könnte. Dies wird vom Arzt als unsinnig abgetan und die Frage als Ausdruck einer übermässigen Angst vor der Operation interpretiert. Er spricht die Patientin ohne Rückfrage, was hinter ihrer Frage stecke, auf ihre psychische Belastungsfähigkeit an; ob sie durch die bevorstehende Menopause in seelische Schwierigkeiten gerate; wenn ja brauche sie vielleicht seelische Unterstützung nach der Operation, die aber trotz ihrer Angst indiziert sei wegen der Grösse des Myoms. Die Patientin ist frustriert, holt sich die Information anderswo, lässt sich operieren, aber von einem anderen Arzt, und versucht in Zukunft, weitere Arztbesuche wo möglich zu vermeiden. Sie hat die Frage als Grenzüberschreitung erlebt, als Lächerlichmachen eines ihr ganz normal erscheinenden Informationsbedürfnisses.

Dies sind zwei Beispiele für missglückte Auftragsklärungen, die Anlass oder Folge einer Beziehungsstörung sein können. Ob nun der Arzt durch seine Unaufmerksamkeit die Beziehungsstörung verursacht oder ob er durch averbale Mitteilungen der Patientin zu diesem Handeln verleitet worden ist, könnte uns der Arzt, der uns den Fall in einer Balintgruppe vorstellt, erzählen.

Mein Balintgruppenmitglied hat mich gefragt, was mich denn nach so langer Zeit Balintgruppenarbeit immer noch fasziniere. Vieles dazu habe ich eigentlich bereits gesagt in meinen Überlegungen $\mathrm{zu}$ der Frage «Was macht denn Angst zu Beginn einer Silser Woche?».
Faszinierend ist, dass es in der Balintgruppenarbeit um ganz grundlegende Dinge geht, die wir in der Alltagssprache beschreiben können, die wir alle kennen, die elementar, erfahrbar, bodenständig sind; wir müssen sie nur wahrzunehmen wagen. Es geht darum wahrzunehmen, wie wir uns fühlen dem Patienten gegenüber, damit wir unsere Beziehung zum Patienten und dessen Beziehung zu uns sehen, reflektieren und wenn nötig klären können. Es geht nicht um «richtige fachspezifische Behandlung». Dies ist wichtiges Thema der verschiedenen Berufe und gehört nicht hierher. Bei ungestörter Beziehung können wir uns als Ärzte z. B. ganz der richtigen Krankheitsbehandlung widmen, als Lehrer der Lehre, als Seelsorger der Seelsorge. Wenn die Beziehung gestört ist, bewirkt die beste Behandlungsabsicht unvorhersehbare, unerwünschte Nebenwirkungen. Die Störung wirkt sich aus auf uns als Person, auf unser Befinden. Wir sind als Personen Wahrnehmungsorgan von Störung. Wir reagieren instinktiv auf eine Diskrepanz zwischen Wort und averbalem Ausdruck unseres Gegenübers, ob wir es wahrnehmen wollen oder nicht. Es wird uns unbehaglich, wir gehen in «Achtung-Vorsicht-Stellung», werden defensiv oder lassen uns unbewusst verführen und fühlen uns high als besserer Partner, bessere Mutter unseres Gegenübers (jedenfalls eine Zeitlang). Um uns als Wahrnehmungsorgan benutzen zu können, müssten wir offen sein - auch uns selber gegenüber, die eigene Reaktion wahrnehmen, dazu stehen, sie als potentiell wichtig betrachten, auch wenn wir sie vorerst nicht verstehen, und wir uns eventuell dafür schämen, sie für unprofessionell usw. halten.

Es braucht auch Mut, diesen Prozess hier in einer gewissen Öffentlichkeit geschehen zu lassen, sich einem Prozess in sich und in der Gruppe zu überlassen, im Vertrauen darauf, dass solche Prozesse eine eigene Richtigkeit haben, und im Vertrauen darauf, dass die Mitspieler wissen, dass wir uns hier persönlich exponieren mit Dingen, die wir sonst eher nicht zeigen. Im Vertrauen darauf, dass die Mitspieler grundsätzlich respektvoll mit uns umgehen, obwohl in diesem Prozess auch ganz unangenehme, bedrohlich scheinende Gefühle aufbrechen und somit in der Gruppe auch Ausdruck finden können, wie Wut, Hass, Verachtung, Ekel, Scham. Wenn der Ausdruck solcher Gefühle möglich ist, ist unser Klima, das wir miteinander schaffen, wahrscheinlich ein günstiges, obwohl das Geschehen manchmal schwer erträglich sein kann. In der Gruppenarbeit ist die «Bedienungsanleitung» für solche Gefühle: sie äussern und dem Gruppenprozess anvertrauen, in welchem sie sich dann 
auch weiterentwickeln können. Wenn sie nach der Gruppenarbeit anhalten, ist es günstig, sich mit andern, die den gleichen Prozess ja auch miterlebt haben, kurzzuschliessen und eher keine Gefühlsabszesse entstehen zu lassen. Im Gruppenprozess (vor allem, wenn dieser erkannt und sichtbar gemacht werden kann - was unsere Hauptaufgabe als Leiter ist) können sich solche Gefühle in Erkennen von Mustern und Verständnis für die Situation des Patienten verwandeln. Zu erleben, dass gerade die mühseligen Gefühle und Verstrickungen uns zu einem besseren und befreienden Verständnis verhelfen, sofern wir diese ernst nehmen und hinschauen, das ist das eigentlich Faszinierende.

Beim Nachdenken über die Angst sind wir beim Faszinierenden gelandet und beim Denken an das Faszinierende bei der Angst. Sind Angst und Faszination zwei Seiten derselben Medaille?

Ich möchte zum Schluss auf mögliche Beziehungsstörungen zu sprechen kommen, die sich mit grosser Wahrscheinlichkeit auch hier in der Seminargruppe einstellen werden. Mit Sicherheit wird es uns nicht - nicht immer - gelingen, eine vorgestellte Beziehung ganz zu verstehen, die unguten Gefühle ganz aufzulösen. Oft ist es so, dass der Referent zwar etwas Wichtiges erkannt hat - es aber für die Gruppe zu wenig klar geworden ist, so dass mühsame Gefühle die Gruppensitzung überdauern. Es wird auch geschehen, dass wir Leiter nicht klar sehen oder allenfalls nur Bruchstücke erkennen. Es kann dann in der Gruppe und auch in der Grossgruppe so etwas entstehen, das wir gut kennen von unserem Umgang mit sogenannt schwierigen
Patienten, die wir nicht (noch nicht) verstehen können: Wir finden sie blöd, unbegabt, unfähig, lästig, zum Davonlaufen - wie auch immer. Der Referent oder andere Gruppenmitglieder, die sich exponiert haben, oder auch der Leiter können sich vorübergehend in der Rolle des schwierigen Patienten wiederfinden (subjektiv oder objektiv) und darunter leiden. Vertrauen wir auf den Prozess und glauben wir daran, dass es weitergeht, vor allem, wenn wir keine «Gefühlsabszesse» entstehen lassen und wir uns mitteilen. Erfahrungsgemäss ist es keine Katastrophe, wenn wir unvollkommen sind und nicht alles verstehen und lösen können - dieser Prozess kann hier eine Woche lang weitergehen -, sei es mit den neu vorgestellten Fällen, die etwas vom Ungelösten wieder aufnehmen, sei es in den Abenddiskussionen, sei es in den informellen Gesprächen zwischendurch.

Dieser sich über eine Woche entwickelnde Gruppenprozess bietet eine zusätzliche Chance: Wir können die Gruppendynamik unter den Teilnehmern, zwischen Leitern und Teilnehmern und unter den Leitern, als weitere Spiegelungsebene der von der Reihe der Fälle ausgelösten Problematik betrachten. So können wir einerseits diese noch klarer verstehen und andererseits fühlen wir uns in diesem Gruppenprozess ganz aktuell und persönlich involviert. Dies bietet die Chance, einiges über sich selber und seine Neigung, so und nicht anders zu reagieren, zu sehen und ein Stück Selbsterfahrung mitzunehmen. Dies gilt für Leiter wie Teilnehmer.

Ich wünsche faszinierendes Arbeiten im mutigen Umgang mit der Angst. 